\title{
Development and experimentation towards a multicast-enabled Internet
}

\author{
D. Careglio*, D. Papadimitriou ${ }^{\dagger}$, F. Agraz*, S. Sahhaf ${ }^{\ddagger}$, J. Perelló*, W. Tavernier ${ }^{\ddagger}$, S. Spadaro*, D. Colle ${ }^{\ddagger}$ \\ ${ }^{*}$ CCABA - Universitat Politécnica de Catalunya, Barcelona, 08034 Spain \\ ${ }^{\dagger}$ Alcatel-Lucent Bell Labs, Antwerp, Belgium

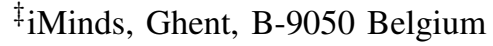

\begin{abstract}
In this paper, we report our development experience and experimentation studies of two multicast routing schemes for the Internet, namely, PIM-SSM and GCMR. We detail their implementation over the Quagga open source routing suite, as well as their experimentation tests over a large-scale topology that reproduces the Internet characteristics.
\end{abstract}

\section{INTRODUCTION AND MOTIVATION}

Although the current Internet does work and is still capable of fulfilling its current missions, it suffers from a relative ossification [1], a condition where any technological innovation meets natural resistance, as exemplified by the lack of widescale deployment of technologies such as multicast or IPv6. Recent initiatives, like the Global Environment for Network Innovations $^{1}$ (GENI), a project sponsored by the National Science Foundation (NSF), and the Future Internet Research and Experimentation ${ }^{2}$ (FIRE) in Europe, try to overcome this ossification problem by bridging the gap between visionary research and technology deployment. The rationale behind them is to promote the experimental validation of new proposals in large-scale realistic network environments, as a way to convince both industry and regulators that a new technology deserves to be adopted and thus enable a possible migration path for technological developments.

Multicast becomes an excellent example of the Internet ossification. Originally defined in the 90 s, its potential benefits have been verified by studies several times since then [2]. However, only intra-domain multicast has been partially adopted in the context of IPTV in some Internet Service Provider (ISP) networks, while inter-domain multicast is still pending to date. Among other reasons, this failure could be attributed to the scaling limitations and relative complexity of the standard multicast protocol architecture, based on overlaying multicast routing on top of the unicast routing topology [3]. For this very reason, we recently proposed the Greedy Compact Multicast Routing (GCMR) scheme [4]. GMCR is characterized by its independence from any underlying unicast routing topology; more specifically, the local knowledge of the cost to direct neighbor nodes is enough for the GCMR scheme to properly operate.

In this paper, we present a prototype of the GCMR multicast scheme and evaluate its functionality and performance on the iLab.t virtual wall ${ }^{3}$ platform, which is a large-scale experimental Linux machine-based emulation testbed. The

\footnotetext{
${ }^{1}$ http://www.geni.net

${ }^{2}$ http://www.ict-fire.eu/

${ }^{3}$ http://www.iminds.be/en/develop-test/ilab-t/virtual-wall
}

prototype of a GCMR routing engine has been developed using the libraries of the Quagga open source routing suite ${ }^{4}$. The success in our endeavor, which was presented during the Hands-on-FIRE! event (collocated in the 2013 FIA Week in Dublin, Ireland), suggests a feasible multicast-enabled Internet.

The rest of the paper is organized as follows. Section II presents a brief overview of the main issues of the current Internet routing system and explains the state of the art in multicast routing. Section III introduces the details of the routing software implementation while Section IV describes the testbed platform. In Section V, we provide and discuss the results. Finally, Section VI concludes the paper.

\section{BRIEF OVERVIEW OF ROUTING AND MULTICAST ROUTING IN INTERNET}

The most fundamental issues faced by the Internet architecture are the scalability, convergence, and stability properties of its inter-domain routing system [5]. Solving them requires to address multiple dimensions together: i) the routing table size growth resulting from a larger number of routing entries, and ii) the routing system dynamics characterized by the routing information exchanges resulting from topological or policy changes. Worst-case projections predict that routing engines could have to process and maintain in the order of 1 million active routes within the next 5 years ${ }^{5}$. Thus, while the Internet routing system prevents from any host specific routing information processing and maintenance (routing state), storing an increasingly large amount of network states in the routing system is expensive and places undue cost burdens on network administrative units. For example, realizing the vision of the Internet of Things, with its corresponding increase on the number of routes, would require significantly more efficient and scalable routing schemes. Furthermore, those impacts on the routing system dynamics (robustness/stability and convergence) resulting from inconsistencies (software implementation errors, router misconfigurations, etc.), instabilities (interactions between routing policies), and topological changes/failures are progressively becoming key concerns for the Internet operational community.

These issues are even more evident if we consider multicast routing system. By multicast routing, we refer to a distributed algorithm that, given a group identifier, allows any node to route multicast traffic to a group of destination nodes, usually called multicast group. To enable one-to-many

\footnotetext{
${ }^{4}$ http://www.nongnu.org/quagga/

${ }^{5}$ http://bgp.potaroo.net/index-bgp.html
} 
traffic distribution, the multicast routing protocol configures the involved routers to build a (logical) delivery tree between the source and the multicast group, commonly referred to as the Multicast Distribution Tree (MDT). Multicast is (re)gaining interest given the increasing popularity of multimedia streaming/content traffic and the explosion of cloud services, since it yields bandwidth savings competing with or complementing cached content distribution techniques [2]. Nevertheless, the scaling problems faced in the 90's still remain mostly unaddressed. Although routing protocol independent routing schemes such as Protocol Independent Multicast (PIM) and Core Base Trees (CBT) [7] have been standardized during last decade, only the Sparse Mode (SM) [6] and the Single Source Multicast (SSM) [8] variant of PIM (PIM-SM and PIMSSM, respectively) has been deployed in the context of IPTV systems for routing multicast streams between VLANs, subnets or access networks (intra-domain multicast) [9]. However, the adoption of inter-domain multicast has failed, as it relies on an overlay routing executing on top of the unicast routing topology, which suffers from the same scaling limits as unicast routing plus the following issues: i) the level of indirection added by the multicast routing as routers forward multicast datagrams to multicast group, and hosts have to subscribe to that multicast group; ii) the limits of shared trees between domains; iii) its address space structure as firewalls have to be upgraded to recognize multicast addresses, iv) management and security complexity, v) the limited number of applications making use of one-to-many connectivity via Internet multicast routing, and many others. A complete analysis of the deployment issues for the IP multicast routing architecture can be found in [3]

In this paper, we experimentally evaluate and compare the performance of the PIM-SSM protocol and the GCMR protocol initially proposed in [4] in the context of interdomain multicast routing. In the following subsections, we describe PIM-SSM and highlight its limitations, which help us to motivate the proposal of GCMR.

\section{A. The protocol independent multicast (PIM) scheme}

PIM [6] is nowadays the most common multicast routing protocols for IP networks that provide one-to-many and manyto-many traffic data distribution. Its protocol-independence comes from the fact that PIM does not perform any networkwide topology discovery mechanism but instead uses routes learned from any unicast routing protocol to build the Multicast Routing Information Base (MRIB), perform the Reverse Path Forwarding (RPF) check, and forward the multicast packets that a router receives from a source. In the context of interdomain routing, Border Gateway Protocol (BGP) has been enhanced with the Multiprotocol BGP (MBGP) extensions [10] to support and distribute IPv6 and multicast addresses.

In PIM-SSM [8], the delivery of traffic data is supported on $(\mathrm{S}, \mathrm{G})$ channels. A $(\mathrm{S}, \mathrm{G})$ channel supports data from the IP unicast source address $S$ to the multicast group address $G$ as the IP destination address. Receivers must subscribe to the $(S, G)$ channel to receive traffic from the specific source $S$. In other terms, a $(S, G)$ channel is the term used in PIM to indicate a MDT. However, applications are responsible of channel discovery. As the PIM scope is limited to routers, the Internet Group Management Protocol (IGMP) in IPv4 or Multicast
Listener Discovery (MLD) in IPv6 needs to be used by hosts (receivers and source) to convey channel subscriptions to local routers. Once a router receives a subscription request from a receiver, it configures the local forwarding table accordingly, and sends the request upstream towards the source address based on its knowledge of the unicast topology. At each hop, routers configure the multicast forwarding table (usually referred as Tree Information Base, TIB) and become members of the MDT (i.e., configuring an entry in the MRIB). At the end of this process, the MDT is built from hosts towards the source, which is considered a shortest-path tree (SPT) from the perspective of the unicast routing tables.

\section{B. The Greedy Compact Multicast Routing (GCMR) scheme}

As part of the work conducted in the EULER FP7-project ${ }^{6}$, we designed the GCMR scheme [4]. Its main objective is to minimize (i.e., to compact) the routing table size at each router by taking local (i.e., greedy) decisions at expenses of i) routing packets on paths with relative small deviation compared to the optimal tree; ii) increasing the number of messages required to create the MDT. In this way, GCMR can reduce the local storage of routing information by keeping only (direct) neighbor-related entries, rather than tree structures or network graph entries. In other terms, the novelty of this algorithm is on maintaining local topology information instead of global one, thus only providing the least cost to next hop during the MDT construction.

Instead of using unicast topology storage information to derive the multicast routing entries (as in the case of all multicast routing protocols such as PIM), in GCMR, the information needed to reach a given multicast source $S$ is acquired by means of a two-stage search process. The algorithm search process is triggered whenever a node decides to join a given multicast source address $S$ as part of a multicast group $G$ or a failure occurs and part of the MDT needs to be restored. This two-stage search process consists of an initial local search covering the receiver neighborhood (defined by a path budget), and, if unsuccessful, a subsequent global search over the remaining topology. The rationale behind this approach is to put tighter limits and search locally first. Indeed, the likelihood of finding any router that belongs to the targeted MDT within a few hops distance from the joining receiver is high in large topologies (whose diameter is logarithmically proportional to its number of nodes) while also increasing with the size of the MDT. Such a search mechanism is triggered whenever a node decides to join a given multicast source address $S$ as part of a multicast group G. Once a node becomes member of an MDT, a multicast routing entry is dynamically created and stored in the local MRIB. From these routing table entries, multicast forwarding entries are also derived and stored in the local TIB. A total of six different types of messages are defined:

- $\quad$ two messages are needed to execute the search process, namely, request (R) and answer (A);

- two messages deal with the logical join (J) and detach (D) of the nodes to/from the MDT;

- $\quad$ a keepalive $(\mathrm{K})$ message is used to check that the link between two elements is operating;

\footnotetext{
${ }^{6}$ http://www.euler-fire-project.eu
} 


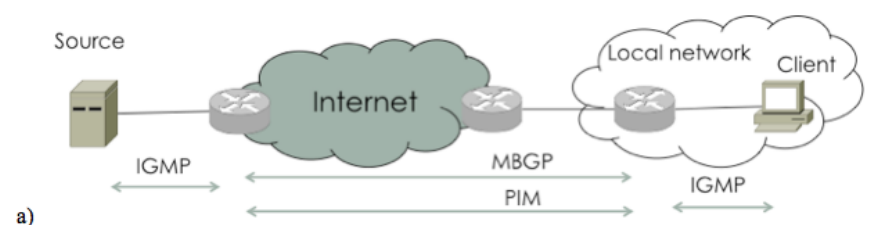

a)

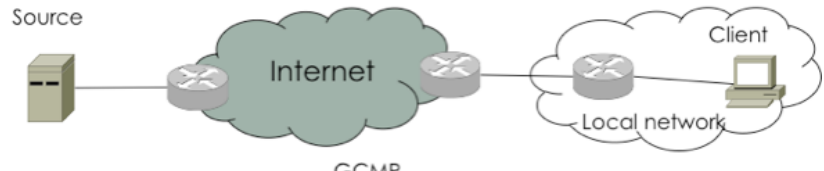

b)

GCMR

Fig. 1. Protocols architecture a) using PIM-SSM, b) using GCMR

- a signalling (S) message is finally sent from source to all clients to detect a failure.

A detailed description of the GCMR algorithm can be found in [11], theoretical performance analysis and simulation comparison with other major multicast routing paradigms are documented in [12].

As GCMR does not rely on any unicast routing protocol, it can work together with any addressing scheme like IPv4, IPv6 or even geometric coordinates. In addition, GCMR can be implemented directly in any host, as its scope is not limited to routers, not requiring any host-router protocol like IGMP. Therefore, to the authors knowledge, it is the first nameindependent, receiver-initiated, dynamic, distributed, end-toend multicast routing algorithm. Finally, although GCMR has been designed to improve scalability of multicast routing in inter-domain environments, it can perform in other environments where routing scalability is also a main issue and only limited topology/routing information is available.

Figure 1a shows the different protocols involved in a typical PIM-SSM scenario and their scope, that is, MBGP for addresses discovery and IGMP for multicast membership subscription between hosts and local routers. In contrast, Figure $1 \mathrm{~b}$ highlights GCMR as a real end-to-end multicast protocol over the Internet.

\section{ROUTING PLATFORM}

As the aim of this work is to experimentally validate GCMR against PIM-SSM, both protocols have been implemented and executed in a prototype. This prototype runs on top of an existing Quagga routing platform (described in this section) in the iLab.t virtual wall emulation testbed platform (Section IV).

\section{A. Quagga routing suite}

Few open source routing platforms exist that allow rapid introduction of new protocols, features, and functionality. Most popular ones are Quagga, $\mathrm{XORP}^{7}$ and $\mathrm{Bird}^{8}$, which can run on standard PC hardware. Among them, we have used Quagga given its widespread adoption and maturity. Quagga benefits from a large developer community including independent code committers, service provides, and academic institutions,

\footnotetext{
${ }^{7}$ http://www.xorp.org

${ }^{8}$ http://bird.network.cz
}

while Bird still presents limitations regarding the inter-domain routing support and XORP is neither mature enough nor widely used for research purposes. Moreover, Quagga starts becoming the common reference platform for software-defined routers running in production environments.

More specifically, Quagga is an open-source routing protocol suite providing implementations of different IP protocols such as OSPF and BGP. The software is developed in standard $\mathrm{C}$ programming language and is available for UNIX platforms like Linux, Solaris and BSD.

The Quagga architecture consists of a core module (i.e., the Zebra daemon), which acts as an abstraction layer to the UNIX kernel packet forwarding functionality. The Zebra daemon provides a set of client modules, called Zserv, implementing each a specific routing protocol. Furthermore, Zebra provides an Application Programming Interface (API), called ZAPI, through which routing protocol modules can access and communicate routing updates to the kernel routing table and network interfaces. During bootstrap, the Zebra daemon must be started first, followed by the routing protocol daemons that should operate in the network. In this way, Zebra is able to interface the communication between the routing daemons and the kernel. For configuration, all daemons in Quagga are equipped with a command-line interface (called VTY), which follows a CISCO OS-like syntax. Alternatively, a pre-defined configuration file can also be used.

It is worth mentioning that neither Quagga nor Bird or even XORP completely emulate a router. They only provide the route engine (algorithms and protocols), thus still requiring a forwarding engine to transmit datagrams. In the following sections, we describe the implementation of GCMR and PIMSSM as well as the forwarding engines used in our prototype.

\section{B. GCMR implementation}

The different modules composing the architecture of a GCMR-capable node are depicted in Figure II-B. The system architecture can be logically divided into two main parts: the Routing Core (RC) and the Routing communication Protocol (RP) module. The former aggregates the set of procedures that a GCMR-capable node must implement in order to carry out its functionalities. The latter module is composed of a set of objects and messages that enables the communication between GCMR nodes and, thus, their collaborative operation.

The RC module contains the main objects that are involved in the GCMR operation. The database contains the information related to the TIB and the MRIB. This module has also two interfaces: one to the RP module and the other enables the communication between the node and an external client (e.g., the application) to trigger the GCMR functionalities, namely create a new multicast group, join the node to a new multicast group and detach the node from an existing multicast group.

The RP module implements the GCMR protocol primitives enabling communication with neighboring nodes and routing operation. Specifically, the protocol relies on two search message types (request $\mathrm{R}$ and answer $\mathrm{A}$ ), which enable the collaborative MDT computation. It also implements two other messages ( $\mathrm{J}$ and D) aimed at the physical join/detach of the nodes to/from the MDT as well messages to failure detection 


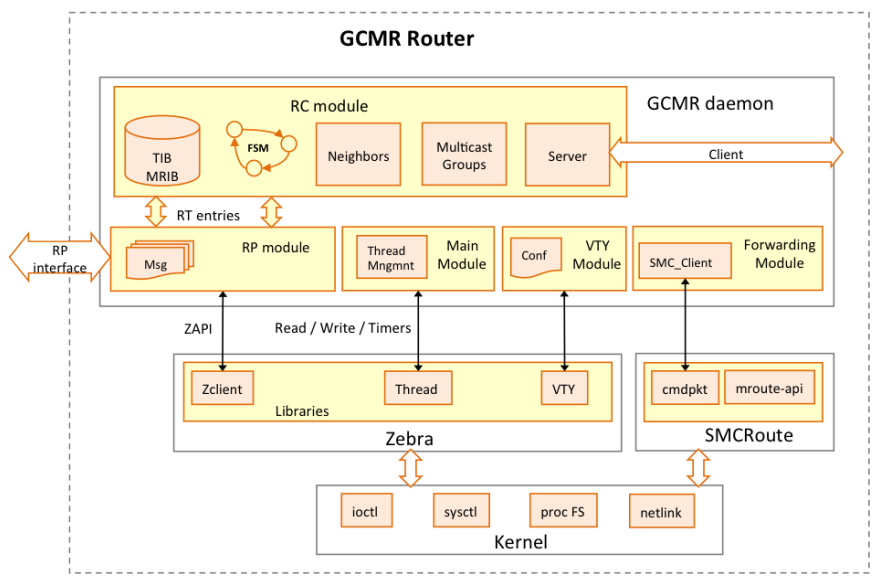

Fig. 2. GCMR node architecture

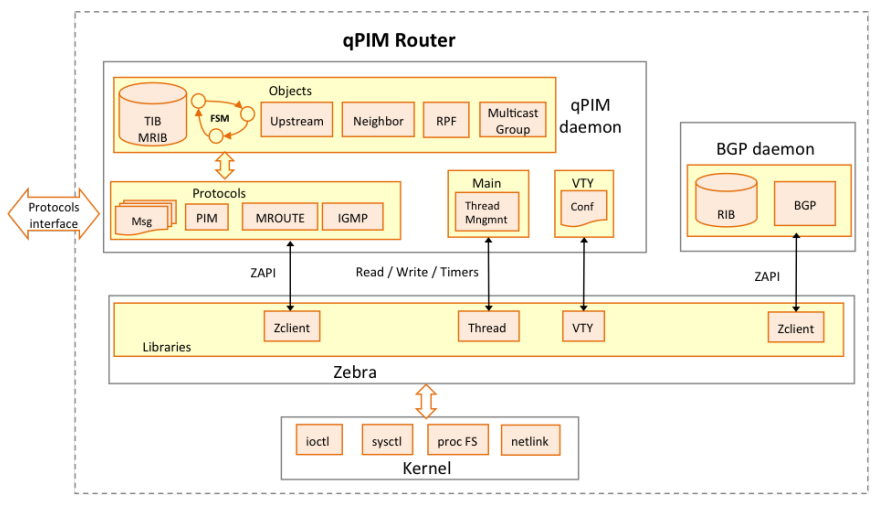

Fig. 3. PIM-SSM node architecture

(S) and localization (K). Through the ZAPI interface, RP communicates with the Zebra daemon in order to configure the interfaces when the node is added or removed to/from a multicast group.

As previously documented, Quagga does not provide any native forwarding engine. For this reason, we have developed an SMCRouteClient that automatically generates multicast route add/remove commands to the SMCRoute daemon ${ }^{9}$. SMCRoute is a command line tool that manipulates dynamic multicast routes directly in the Linux kernel.

\section{PIM-SSM implementation}

An implementation of the PIM-SSM routing protocol, called qPIM ${ }^{10}$, is available as an external daemon for Quagga routing suite. The architectural view of this PIM-capable node is depicted in Fig. 3. The qPIM daemon consists of two modules, namely the Objects module that constructs and maintains the set of data structures and the objects and the Protocols module that implements the communication protocols.

As described in Section II-A, PIM-SSM requires two additional protocols to operate. BGP daemon (with multiprotocol extensions) of Quagga is used as unicast routing protocol to populate the routing table, from which PIM-SSM derives

\footnotetext{
${ }^{9} \mathrm{http}: / / \mathrm{www} . c s c h i l l . d e /$ smcroute/

${ }^{10} \mathrm{http}: / /$ www.nongnu.org/qpimd/
}

the MRIB entries during the MDT construction. The IGMP protocol is used to interact hosts with routers. As a forwarding engine, qPIM uses the conventional ipmroute daemon of the Linux kernel to derive the TIB entries from the MRIB and generate the add/remove commands to configure the network interfaces accordingly.

\section{ILAB.T VIRTUAL WALL PLATFORM}

The iLab.t virtual wall (VW)is a generic test environment, which provides computing hardware and different software and hardware tools to researchers to validate and evaluate the performance of innovative network software prototypes. Each of the $3 \mathrm{VW}$ facilities of iLab.t consists of 100 server blades interconnected by a non-blocking $1.5 \mathrm{~Tb} / \mathrm{s}$ VLAN Ethernet switch (Force 10 E1200). The specification of each node is as follows: dual processor, dual core server with 4GB RAM and $4 \times 80 \mathrm{~GB}$ hard disk with $6 \times 1 \mathrm{~Gb} / \mathrm{s}$ or $4 \times 1 \mathrm{~Gb} / \mathrm{s}$ interfaces. Moreover, a control interface is provided in each node, which enables researchers to login.

Traditional mapping between Linux machines and nodes is one-to-one thus the emulation of network topologies is limited to the number of physical machines. On the iLab.t virtual wall, virtualization technique using OpenVZ ${ }^{11}$ Linux containers allows for multiple virtual nodes to run on one machine enabling large-scale experiments (10-20 times the number of physical machines in the iLab.t). In addition, arbitrary number of virtual network links is provided by using virtual network interfaces. These links may be individually shaped and may be multiplexed over physical links or can be used to connect to virtual nodes within one physical node.

To experimentally validate and compare PIM-SSM and GCMR, we used around 45 server blades of one of the iLab.t virtual wall facility. Each blade has been virtualized into approximately 5 virtual machines in order to reach a topology of more than 200 nodes. In each node, we installed a Debian 6 Linux distribution (kernel 2.6.32) and Quagga 0.99.17. Both PIM-SSM and GCMR were then automatically deployed in the nodes and properly configured using Phyton and bash scripts. Information of the nodes status was continuously stored in log files and processed afterward to obtain the performance results.

\section{EXPERIMENTATION AND DISCUSSION OF THE RESULTS}

\section{A. Experimentation setup and objectives}

The main objective of these tests is to demonstrate the successful operation of GCMR in the context of inter-domain routing over a large-scale network topology compared to the standard PIM-SSM protocol. In particular, the following performance metrics are evaluated:

- $\quad$ stretch, defined as the sum of the weights of edges used in multicasting from the source to all receivers divided by the optimal such tree. Intuitively, the stretch of a routing scheme provides a quality measure of the path cost increase it produces compared to the optimal tree (which has clearly a stretch of 1 ). The benchmarking reference optimal MDT (so-called Steiner Tree [4]) is computed offline knowing the server and all receivers beforehand.

\footnotetext{
${ }^{11}$ http://openvz.org
} 


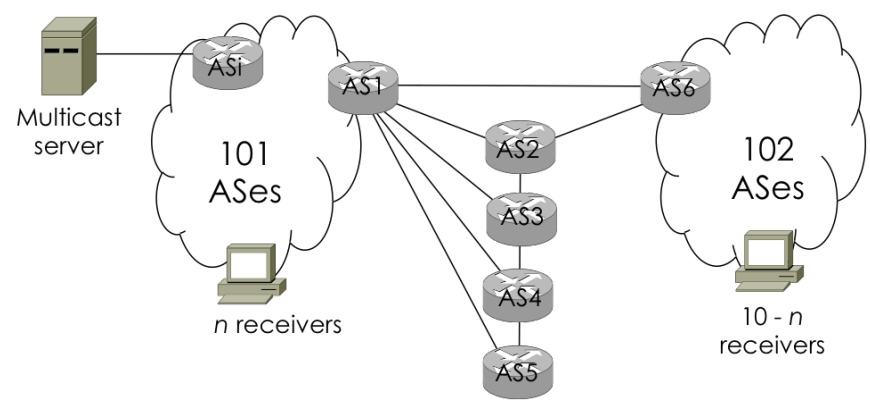

Fig. 4. Topology for the experiments

- $\quad$ routing table (RT) size, defined as the maximum number of memory-bits required to locally store the RT entries. Thus, the RT size is computed using the bit-size of a single entry and the number of entries it comprises. The storage required by the algorithm is directly related to routing system scalability.

- $\quad$ recovery time, defined, in this case, as the maximum time needed to receive back a multicast transmission at the receivers once a failure occurs in a link of the MDT.

- communication cost, is defined as the number of routing updated messages that needs to be exchanged between routers to converge after a change (e.g., a new receiver joins the MDT).

The experimentation tests of PIM-SSM and GCMR were performed in a network consisting of 207 Autonomous Systems (ASes). As we were interested in the inter-domain aspects, we represented the behavior of each AS with a single router with multiple interfaces (Fig. 4). This 207 ASes emulates a portion of the Internet where one AS provides a multicast service to the rest of 206 ASes. In particular, we executed ten runs of the same experiment: one multicast server located in one AS is firstly selected and then ten receivers, located in ten different ASes, joined the MDT sequentially. Both the server and the receivers were randomly chosen.

The considered topology presents a particular structure consisting of AS1-AS6 that interconnects two blocks of ASes. The reason of this AS1-AS6 connectivity is to enforce the so-called path exploration problem of BGP [13]. In fact, path exploration suggests that, in response to path failures or routing policy changes, some BGP routers may try a number of transient paths before selecting a new best path or declaring unreachability to a destination. Consequently, a long time period may elapse before the whole network eventually converges to the final decision, resulting in slow routing convergence. We will analyze this phenomenon when discussing the results.

\section{B. Results and discussions}

Figure 5 shows the stretch of GCMR and PIM-SSM in ten different executions. GCMR presents in all runs lower stretch than PIM-SSM ( 0.14 better on average) and some deterioration ( 0.095 on average) against the optimal MDT (remind that stretch-1 is the reference). Using the information obtained from BGP, PIM-SSM establishes a shortest path tree (SPT) between

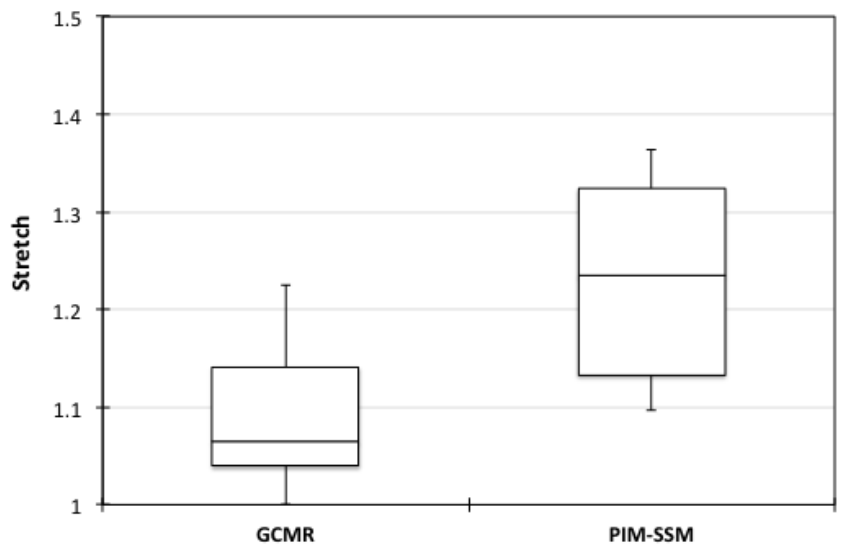

Fig. 5. Comparison of stretch

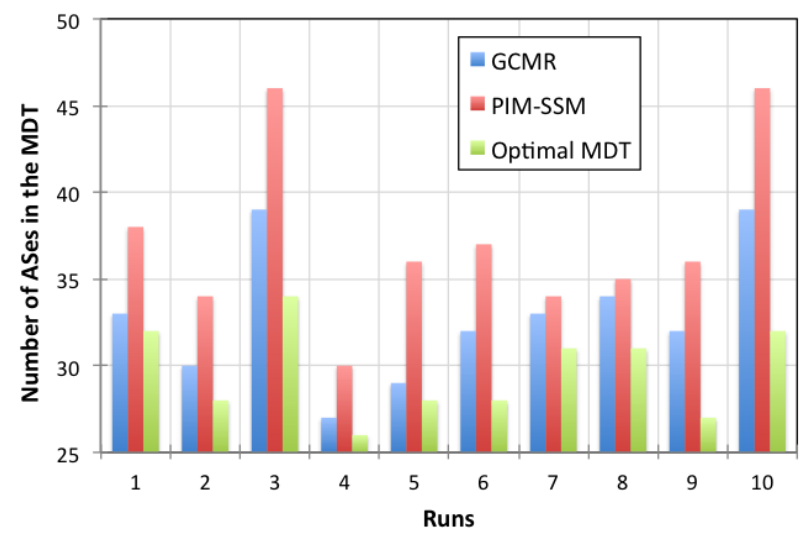

Fig. 6. Number of ASes in the MDT

the receivers and the source. This result is consistent with the simulation results provided in [4], where, in much more larger simulation scenarios ( $32 \mathrm{k}$ nodes) and high number of receivers (500-4000), GCMR obtains approximately 0.1 better stretch than SPT.

In Fig. 6, we show the number of ASes involved in the MDT. As expected from the previous results, GCMR is much more closer to the optimal MDT than PIM-SSM. This is an important outcome of GCMR from the cost point of view as providers charge customers based on the proportion of their resources that are used in multicast. Thus, as GCMR uses less transit ASes, the cost for the customers should be less using GCMR than PIM-SSM.

The comparison in terms of Routing Table (RT) size is presented in Fig. 7. To determine the size of the RT (in bits), we only consider the nodes involved in the MDT and use the RT formats defined in [11]: while GCMR only needs MRIB and TIB information, PIM-SSM, besides MRIB and TIB, also needs some unicast information from BGP to determine the shortest path towards the multicast source. Obtained results show that GCMR requires around $44 \%$ less bits than PIMSSM.

Regarding the recovery time, we emulated a failure in the link connecting AS1 and AS6 and counted the maximum 


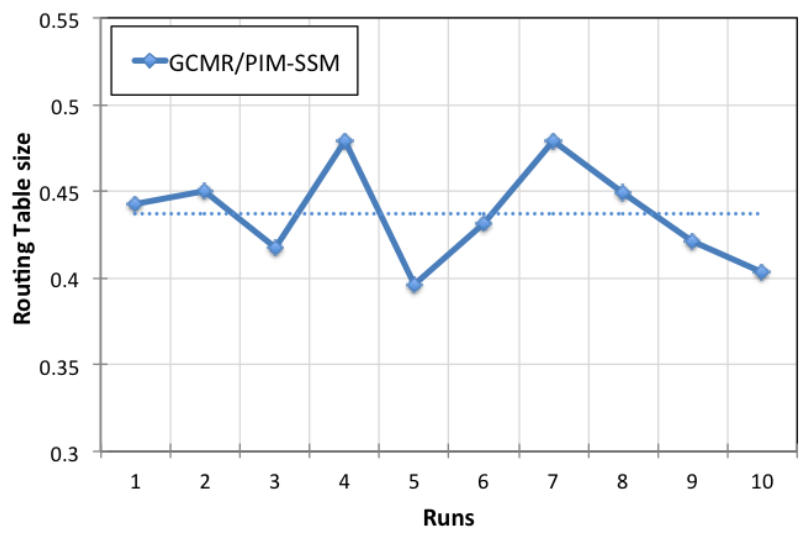

Fig. 7. Comparison of the Routing Table size (in bits)

time elapsed to receive back the multicast transmission at the affected receivers. In the case of PIM-SSM, it equals the number of Minimum Route Advertisement Interval (MRAI) -in seconds- elapsing between the withdraw of the routing state corresponding to the initial route towards the multicast source and the next stable state where BGP routing state convergence can be declared [10] plus the time required for PIM Hello adjacency and Join message exchange with the new next hop router along the route to the multicast source node [8]. Due to the particular structure of AS-AS6 interconnection, BGP tends to explore all alternatives (problem known as path exploration [13]) before reaching a stable state and, as a consequence, the obtained traffic interruption result quite high in the experiments, 2 minutes and half approximately. In the case of GCMR, recovery time comprises the time for the failure-detecting node to initiate a search and receive answers from its neighbors that point to the least cost branching path plus the time to initiate a Join message. In the experiments, the recovery time is of the order of one second.

Finally, we estimate the communication cost of PIM-SSM and GCMR to evaluate the control overhead of the protocols. In the considered scenario, GCMR requires around 20\% more messages than PIM-SSM to setup the MDT.

It is worth mentioning that we compared GCMR and PIMSSM also in larger scenario with an ad-hoc simulator. In particular, we tested them in a network with around 46,000 ASes. Simulation results showed similar trends than emulation results. For instance, to setup an MDT of 4000 receivers, GCMR obtains better stretch, very quick recovery time and two order of magnitude smaller routing tables than PIM at the expense of higher communication cost (around 50 times more).

\section{CONCLUSIONS}

This paper experimentally validates and compares the performance of standardized PIM-SSM multicast routing algorithm, which uses BGP for path discovery, and the novel GCMR compact multicast routing scheme recently proposed. In a large-scale experimental facility, the GCMR scheme provides a better performance compared to PIM-SSM in terms of the memory space it requires to locally store the routing information and the stretch factor increase multicast routing paths it produces. Moreover, the adaptive property of GCMR induces a limited number of re-routing events in case of failure compared to the recovery strategy applied by the combination of BGP and PIM-SSM.

Regarding future work, we will focus on reducing the (current high) communication cost of GCMR implementing better search engine and/or adapting GCMR to the any-source multicast method [6]. Future experimental demonstration aims at validating GCMR in even larger topology (e.g., O(10k) ASes) with multiple concurrent multicast sessions [14]. Finally, the good results obtained with GCMR motivate a future analysis of its possible deployability in real environments; as Internet cannot be considered an option at this stage of development, another particular environments where routing scalability is also a main issue and only limited topology/routing information is available will be considered first.

\section{ACKNOWLEDGMENT}

This work has been partially funded by the EULER (FP7258307) and DOMINO (TEC2010-18522) projects.

\section{REFERENCES}

[1] J.S.Turner, D.E.Taylor, "Diversifying the Internet", in Proc. IEEE Globecom 2005, St. Louis, MO, USA, Dec. 2005

[2] S. Ratnasamy, A. Ermolinskiy, S. Shenker, "Revisiting IP multicast", in Proc. Sigcomm '06, Pisa, Italy, pp. 15.26, Sep. 11-15, 2006.

[3] C. Diot, et al., "Deployment Issues for the IP Multicast Service and Architecture", IEEE Network, vol. 4, no. 1, pp. 78-88, Jan. 2000.

[4] P. Pedroso, D. Papadimitriou, D. Careglio "Dynamic compact multicast routing on power-law graphs", in Proc. IEEE Globecom 2011, Houston, TX, USA, Dec. 2011.

[5] T.Li (ed.), "Design Goals for Scalable Internet Routing", IETF RFC 6227, May 2011.

[6] B. Fenner et.al., "Protocol Independent Multicast - Sparse Mode (PIMSM)", IETF RFC 4601, Aug. 2006.

[7] T. Ballardie, P. Francis, J. Crowcroft, "Core Based Trees (CBT): An Architecture for Scalable Multicast Routing", in Proc. ACM Sigcomm 1995, Cambridge, MA, USA, Aug. 1995.

[8] H. Holbrook, B. Cain, "Source-Specific Multicast for IP", IETF RFC 4607, Aug. 2006

[9] L. Zheng, J. Zhang, R. Parekh, "Survey Report on Protocol Independent Multicast - Sparse Mode (PIM-SM) Implementations and Deployments", IETF RFC 7063, Dec. 2013.

[10] T. Bates, et al., "Multiprotocol Extensions for BGP-4", IETF RFC 4760, Jan. 2007.

[11] P. Pedroso, D. Papadimitriou, D. Careglio, "A name-independent compact multicast routing algorithm", Technical Report, UPC-DAC-RRCBA-2011-15, Mar. 2011

[12] D. Papadimitriou, D. Careglio, P. Demeester, "Performance analysis of multicast routing algorithms", in Proc. ICNC '14, Honolulu, HA, USA, Feb. 3014.

[13] R. Oliveira, B. Zhang, Dan Pei, Lixia Zhang, "Quantifying Path Exploration in the Internet", IEEE/ACM Trans. Net., vol. 17, no. 2, Apr. 2009.

[14] D. Careglio, D. Papadimitriou, F. Agraz, S. Sahhaf, J. Perell, W. Tavernier, "On the experimentation of the novel GCMR multicast routing in a large-scale testbed", in Proc. 33rd Annual IEEE Infocom 2014 - demo session, Toronto, Canada, Apr. 27, 2014. 